\title{
A Theorem on Single-Peaked Preference Functions in One Dimension
}

\author{
Clyde H. Coombs* and George Avrunin
}

Department of Psychology, The University of Michigan, Ann Arbor, Michigan 48109

With simple stimuli like amount of sugar in coffee or grade expectations in courses, the preference orders of individuals can be represented by single-peaked functions of an underlying ordering. When stimuli are more complex, as in candidates for office or automobiles, there is usually no natural ordering underlying the stimuli, and hence the preference orders cannot be single-peaked functions in one dimension.

Is the existence of an underlying ordering necessary and sufficient to ensure that all preference orders will be single-peaked and if not, just what further conditions must be met? An understanding of the precise mathematical conditions will be useful in the interpretation of preferential choice data and will show experimenters how to essentially guarantee that all subjects will be single-peaked.

The theorem presented here is essential to an understanding of the intimate relation between single-peaked functions and preferential choice behavior and deals with the general case of options which may differ in more than two components. The application of the theorem to the special cases of options which differ in only one or two components has been extensively developed and has appeared elsewhere (Coombs \& Avrunin, 1977).

Given a set of options $S$, we will assume that each attribute of the options in $S$ which is relevant to the decision process, whether it be a physical, psychological, or social attribute, can be expressed in terms of a numerical scale. We can then represent each option in $S$ as a vector, each component of which corresponds to a particular attribute of the options. Thus we can regard $S$ as a subset of $\mathbb{R}^{n}$ for some $n$ depending on $S$ and the decision process (the latter determines what attributes are relevant).

We assume that on each component of $\mathbb{R}^{n}$ there is a proper utility function defined as follows.

Definition. A proper utility function has second derivative everywhere negative.

If the function is monotone-increasing with the component we call it a "good" or attractive attribute, and if it is monotone-decreasing we call it a "bad" or unattractive attribute. These are the classical marginally decreasing utility functions of economics.

Among the components relevant to the decision process for a particular set of options, some will be good and some will be bad, or all will be good or all will be bad. In the first case of some good attributes and some bad, the context of the decision is an approach-

* This research was supported by National Science Foundation under Grant GB-41877 to the University of Michigan. 
avoidance conflict, the other two cases are respectively referred to as an approachapproach conflict and an avoidance-avoidance conflict.

Single-peakedness in one dimension is defined only with respect to a given ordering of the options, as follows:

Definition. A preference function is single-peaked in one dimension if $A<B<C$ implies that the preference ordering is neither $A>_{p} C>_{p} B$ nor $C>_{p} A>_{p} B$, i.e., the intermediate option is never least preferred.

Given a preference function and any set of options, we may order the options in such a way as to make the preference function single-peaked; consequently, we need to define an ordering independently of the preference function. But there is no natural ordering of a general set of options in the multiattribute case, so we restrict our attention to special sets of options which we will call efficient sets. We define the concept of an efficient set in such a way as to ensure the cxistcnce of a unidimensional single-peaked preference function over the set in the presence of proper utility functions and under a very general rule of composition for the preference function, defined subsequently.

Definition. An approach-avoidance efficient set in $n$ dimensions $(n \geqslant 2)$ is a subset $S$ of $\mathbb{R}^{n}$ together with a partition of the integers, $1,2, \ldots, n$ into two nonempty subsets $I_{1}$ and $I_{2}$, such that the following conditions are satisfied:

(1) For any pair $\left(x_{1}, \ldots, x_{n}\right)$ and $\left(y_{1}, \ldots, y_{n}\right)$ in $S$, (i) $x_{i} \leqslant y_{i}$ for some $i$ iff $x_{j} \leqslant y_{j}$ for all $j=1, \ldots, n$ and (ii) $x_{i}<y_{i}$ for some $i \in I_{1}$, iff $x_{j}<y_{j}$ for some $j \in I_{2}$.

(2) For each triple $\left(x_{1}, \ldots, x_{n}\right),\left(y_{1}, \ldots, y_{n}\right),\left(z_{1}, \ldots, z_{n}\right)$ in $S$ with $x_{1}<y_{1}<z_{1}$, there exists a $c>0$ such that

$$
\begin{array}{ll}
c\left(y_{i}-x_{i}\right) \geqslant z_{i}-y_{i} & \text { for all } i \in I_{1}, \\
c\left(y_{j}-x_{j}\right) \leqslant z_{j}-y_{j} & \text { for all } j \in I_{2} .
\end{array}
$$

In an approach-avoidance efficient set, we think of the components indexed by elements of $I_{1}$ as the "good" components and those indexed by $I_{2}$ as the "bad" components. If the options of $S$ have attributes with both good and bad aspects, we can imbed $S$ in a higher-dimensional space to achieve this.

The first condition in the definition is an extension of the property of Pareto optimality in $n$ dimensions. A natural extension of Pareto optimality merely ensures nondomination of one option by another. This extension goes further: it ensures that the ordering on all components is the same so any one may be used to define an ordering on the options, and also ensures that if one option is better than another on a good component it will be worse than the other on a bad component. The second condition asserts the existence of a constant $c>0$ with the property that the largest proportionate increment in the good components is no greater than $c$ and the smallest proportionate increment in any bad component is no less than $c$.

This notion of efficiency implies that the options lie on the boundary of their convex hull but is actually a stronger condition in $\mathbb{R}^{n}$ for $n \geqslant 3$. 
We may reduce the other two cases to an approach-avoidance conflict as follows:

Definition. An approach-approach efficient set in $n$ dimensions $(n \geqslant 2)$ is a subset $S$ of $\mathbb{R}^{n}$ together with a partition of the integers $1,2, \ldots, n$ into two nonempty subsets $I_{1}$ and $I_{2}$ so that, if we set $\epsilon_{i}$ equal to 1 or -1 according as $i$ is in $I_{1}$ or $I_{2}$, the set $\left\{\left(\epsilon_{1} x_{1}, \ldots, \epsilon_{n} x_{n}\right) \mid\left(x_{1}, \ldots, x_{n}\right) \in S\right\}$ with $I_{1}$ and $I_{2}$ is an approach-avoidance efficient set.

Thus, an approach-approach efficient set satisfies the conditions:

(1) for any pair $\left(x_{1}, \ldots, x_{n}\right)$ and $\left(y_{1}, \ldots, y_{n}\right)$ in $S$, (i) $x_{i} \leqslant y_{i}$ for some $i \in I_{1}$, iff $x_{j} \leqslant y_{j}$ for all $j \in I_{1}$, and $x_{k} \geqslant y_{k}$ for all $k \in I_{2}$, while $x_{i} \geqslant y_{i}$ for some $i \in I_{2}$ iff $x_{j} \leqslant y_{j}$ for all $j \in I_{1}$ and $x_{k} \geqslant y_{k}$ for all $k \in I_{2}$, and (ii) $x_{i}<y_{i}$ for some $i \in I_{1}$ iff $x_{j}>y_{j}$ for some $j \in I_{2}$.

(2) for each triple $\left(x_{1}, \ldots, x_{n}\right),\left(y_{1}, \ldots, y_{n}\right),\left(z_{1}, \ldots, z_{n}\right)$ in $S$ with $x_{i}<y_{i}<z_{i}$ for some (and hence all) $i \in I_{1}$, there exists $c>0$ so that $c\left(y_{j}-x_{j}\right) \geqslant z_{j}-y_{j}$ for all $j=1, \ldots, n$.

Heuristically, an approach-approach efficient set with three attributes, all desirable, is one in which two of the attributes must increase (decrease) together in the same rank order over the options and the third must decrease (increase) in the reverse order. This is the property of Pareto optimality and creates the conflict between more of one good(s) at the cost of less of another good(s). The existence of a $c>0$ has exactly the same interpretation as given for the definition of an approach-avoidance set. Of course, if all the attributes are desirable, the semantics of $I_{1}$ and $I_{2}$ are different. Increases on attributes represented by components in $I_{1}$ are necessarily accompanied by decreases on attributes represented by components in $I_{2}$.

Definition. An avoidance-avoidance efficient set in $n$ dimensions $(n \geqslant 2)$ is, similarly, a subset $S$ of $\mathbb{R}^{n}$ together with a partition of the integers $1,2, \ldots, n$ into two nonempty subsets $I_{1}$ and $I_{2}$ so that, if we set $\delta_{i}$ equal to -1 or +1 according as $i$ is in $I_{1}$ or $I_{2}$, the set $\left\{\left(\delta_{1} x_{1}, \ldots, \delta_{n} x_{n}\right) \mid\left(x_{1}, \ldots, x_{n}\right) \in S\right\}$ with $I_{1}$ and $I_{2}$ is an approach-avoidance efficient set.

Thus an avoidance-avoidance efficient set satisfies exactly the same conditions as an approach-approach efficient set except for the semantics associated with $I_{1}$ and $I_{2}$.

Suppose that $E=\left(S, I_{1}, I_{2}\right)$ is an approach -avoidance efficient set with $I_{1}$ the sct of components representing attractive attributes of the options of $S$ and with $I_{2}$ the set of components representing unattractive attributes, or that $E$ is an approach-approach or avoidance-avoidance efficient set. Then if $\phi_{1}, \ldots, \phi_{n}$ are proper utility functions, it is clear from the definitions that the set $\left\{\left(\phi_{1}\left(x_{1}\right), \ldots, \phi_{n}\left(x_{n}\right)\right) \mid\left(x_{1}, \ldots, x_{n}\right) \in S\right\}$, together with $I_{1}$ and $I_{2}$, is an efficient set of the same type as $E$. In other words, efficiency is preserved under this kind of transformation induced by the proper utility functions.

We now introduce a composition rule for the preference function.

Definition. We say that a function $F: \mathbb{R}^{n} \rightarrow \mathbb{R}$ is a proper preference function for a set of options $S \subseteq \mathbb{R}^{n}$ if: 
(1) all its first-order partial derivatives $\partial F / \partial u_{i}$ exist, where the $u_{i}$ are the coordinate functions,

(2) $x_{j} \leqslant y_{j}$ for all $j$ and $x_{i}<y_{i}$ implies

$$
\frac{\partial F}{\partial u_{i}}\left(x_{1}, \ldots, x_{n}\right) \geqslant \frac{\partial F}{\partial u_{i}}\left(y_{1}, \ldots, y_{n}\right) \geqslant 0
$$

when the $i$ th component represents an attractive attribute,

(3) $x_{j} \leqslant y_{j}$ for all $j$ and $x_{i}<y_{i}$ implies

$$
0 \geqslant \frac{\partial F}{\partial u_{i}}\left(x_{1}, \ldots, x_{n}\right) \geqslant \frac{\partial F}{\partial u_{i}}\left(y_{1}, \ldots, y_{n}\right)
$$

when the $i$ th component represents an unattractive attribute.

So, if the partial derivatives of a preference function form a set of proper utility functions, then the function is a proper preference function. Another way of putting it is that a proper preference function is marginally decreasing in every component independent of increases in any other component. For example, if $\phi_{1}, \ldots, \phi_{n}$ are proper utility functions and $a_{1}, \ldots, a_{n}$ are positive real numbers, then $F\left(x_{1}, \ldots, x_{n}\right)=\sum_{i=1}^{n} a_{i} \phi_{i}$ is a proper preference function.

The property of single-peakedness is preserved under strictly increasing transformations of a preference function, but of course, all such transformations of a preference function need not be proper preference functions. Given any preference function $G$, we show in the theorem below that if there exists one strictly increasing transformation, $g$, for which the preference function $g \circ G$ is proper, then $G$ is single-peaked on an efficient set and all preference functions generated from it by strictly increasing transformation are, of course, single-peaked.

THEOREM. Suppose that $E=\left(S, I_{1}, I_{2}\right)$ is an approach-avoidance efficient set in $n$ dimensions with $I_{1}$ the set of components representing attractive attributes of the options of $S$ and with $I_{2}$ the set of components representing unattractive attributes, or that $E$ is an approach-approach or avoidance-avoidance efficient set. If $G: \mathbb{R}^{n} \rightarrow \mathbb{R}$ is a function such that for some strictly increasing $g: \mathbb{R} \rightarrow \mathbb{R}, F=g \circ G$ is a proper preference function for $S$, then $G$ is single-peaked on $E$ with respect to the ordering by values on the components of either $I_{1}$ or $I_{2}$.

Proof. We will give the proof only for the case where $E$ is an approach-avoidance efficient set. The arguments in the other cases are similar.

Since $g$ preserves order it suffices to prove that $F$ is single-peaked on $E$. To simplify the notation, assume $I_{1}=\{1, \ldots, m\}$ and $I_{2}=\{m+1, \ldots, n\}$. Suppose $\left(x_{1}, \ldots, x_{n}\right)$ and $\left(y_{1}, \ldots, y_{n}\right)$ and $\left(z_{1}, \ldots, z_{n}\right)$ are options in $S$ with $x_{1}<y_{1}<z_{1}$ (in this case the orderings are the same on each component), and $F\left(x_{1}, \ldots, x_{n}\right)>F\left(y_{1}, \ldots, y_{n}\right)$. We will show $F\left(y_{1}, \ldots, y_{n}\right)>F\left(z_{1}, \ldots, z_{n}\right)$. 
By repeated application of the mean value theorem, we find that

$$
\begin{aligned}
F\left(y_{1}, \ldots, y_{n}\right)= & F\left(x_{1}, \ldots, x_{n}\right)+\sum_{i \in I_{1}} \frac{\partial F}{\partial u_{i}}\left(y_{1}, \ldots, y_{i-1}, a_{i}, x_{i+1}, \ldots, x_{n}\right) \\
& \cdot\left(y_{i}-x_{i}\right)+\sum_{j \in I_{2}} \frac{\partial F}{\partial u_{i}}\left(y_{1}, \ldots, y_{j-1}, a_{j}, x_{j+1}, \ldots, x_{n}\right) \cdot\left(y_{j}-x_{j}\right),
\end{aligned}
$$

where $a_{k}$ is some number between $x_{k}$ and $y_{k}$ for $k=1, \ldots, n$ and the $U_{i}$ are the coordinate functions.

Since $F\left(y_{1}, \ldots, y_{n}\right)<F\left(x_{1}, \ldots, x_{n}\right)$, we know that the sum of the two terms involving partial derivatives is negative.

In the same fashion, we get

$$
\begin{aligned}
F\left(z_{1}, \ldots, z_{n}\right)= & F\left(y_{1}, \ldots, y_{n}\right)+\sum_{i \in I_{1}} \frac{\partial F}{\partial u_{i}}\left(z_{1}, \ldots, z_{i-1}, b_{i}, y_{i+1}, \ldots, y_{n}\right) \\
& \cdot\left(z_{i}-y_{i}\right)+\sum_{j \in I_{2}} \frac{\partial F}{\partial u_{j}}\left(z_{1}, \ldots, z_{j-1}, b_{j}, y_{j+1}, \ldots, y_{n}\right) \cdot\left(z_{j}-y_{j}\right),
\end{aligned}
$$

where $b_{k}$ is some number between $y_{k}$ and $z_{k}$ for $k=1, \ldots, n$. For $i \in I_{1}$, we know $y_{i}-x_{i} \geqslant c\left(z_{i}-y_{i}\right)$ where $c>0$ because $E$ is an efficient set and, since $b_{i}>a_{i}$, we know

$$
\frac{\partial F}{\partial u_{i}}\left(y_{1}, \ldots, y_{i-1}, a_{i}, x_{i+1}, \ldots, x_{n}\right) \geqslant \frac{\partial F}{\partial u_{i}}\left(z_{1}, \ldots, z_{i-1}, b_{i}, y_{i+1}, \ldots, y_{n}\right)
$$

so we have

$$
\begin{aligned}
\sum_{i \in I_{1}} & \frac{\partial F}{\partial u_{i}}\left(y_{1}, \ldots, y_{i-1}, a_{i}, x_{i+1}, \ldots, x_{n}\right) \cdot\left(y_{i}-x_{i}\right) \\
& \geqslant c \sum_{i \in I_{1}} \frac{\partial F}{\partial u_{i}}\left(z_{1}, \ldots, z_{i-1}, b_{i}, y_{i+1}, \ldots, y_{n}\right) \cdot\left(z_{i}-y_{i}\right) \\
& \geqslant 0 .
\end{aligned}
$$

Similarly, we have

so

$$
\begin{aligned}
0 & \geqslant \sum_{j \in I_{2}} \frac{\partial F}{\partial u_{j}}\left(y_{1}, \ldots, y_{j-1}, a_{j}, x_{j+1}, \ldots, x_{n}\right) \cdot\left(y_{j}-x_{j}\right) \\
& \geqslant c \sum_{j \in I_{2}} \frac{\partial F}{\partial u_{j}}\left(z_{1}, \ldots, z_{j-1}, b_{j}, y_{j+1}, \ldots, y_{n}\right) \cdot\left(z_{j}-y_{j}\right),
\end{aligned}
$$

$$
\begin{aligned}
& \sum_{i \in I_{1}} \frac{\partial F}{\partial u_{i}}\left(z_{1}, \ldots, z_{i-1}, a_{j}, y_{i+1}, \ldots, y_{n}\right) \cdot\left(z_{i}-y_{i}\right) \\
& \quad+\sum_{j \in I_{2}} \frac{\partial F}{\partial u_{j}}\left(z_{1}, \ldots, z_{j-1}, b_{j}, y_{i+1}, \ldots, y_{n}\right) \cdot\left(z_{j}-y_{j}\right)
\end{aligned}
$$


must also be negative, and thus,

$$
F\left(y_{1}, \ldots, y_{n}\right)>F\left(z_{1}, \ldots, z_{n}\right),
$$

and $F$ is single-peaked on $E$ as claimed.

Q.E.D.

If the set $S$ is not efficient, it is easy to construct a proper preference function which is not single-peaked on $S$ even if $S$ is Pareto optimal. Thus, although some proper preference functions may be single-peaked on a nonefficient set, the property of efficiency is necessary and sufficient to ensure that all proper preference functions be single-peaked.

In summary, given that individuals have proper preference functions, then a necessary and sufficient condition for all preference functions to be single-peaked is that the set of options be an efficient set. If the options are complex in the sense of differing on three or more components, then a necessary and sufficient condition for an efficient set is that the options be same-ordered (or reversed) on all components, and that the proportionate changes on any good component can never overcome the proportionate changes in any bad component so that the coordinates of the options on any two components, one a good one and the other a bad one, will be an efficient set in two dimensions.

In the case that the options differ in only one component, the set is inevitably efficient, and all preference orders will be single-peaked under proper preference functions. If the options differ in exactly two dimensions, then nondomination (Pareto optimality) will ensure that the options are same-ordered (or reversed) on both components, and hence, approximates an efficient set and "most" preference functions will be single-peaked. If the options differ on three or more components, an efficient set would have to be contrived; otherwise, preference orders will not necessarily be single-peaked.

This theorem shows experimenters how they can ensure that subjects' preference orders will be single-peaked over a set of complex stimuli, for example, in the study of psychological risk where gambles or lotteries can vary in several dimensions. These results also have implications for multidimensional unfolding and for conflict resolution, as discussed elsewhere (Coombs \& Avrunin, 1977).

\section{REFERENCE}

Coombs, C. H., \& Avrunin, G.S. Single-peaked functions and the theory of preference. Psychological Review, 1977, 84, 216-230.

ReCeIVED: July 28, 1976 